\title{
A Case Report of Herpes Zoster
}

\author{
Dr.ParulkarGeeta D., M.D.(Chikitsa)(Mum),PhD(Chikitsa)(Mum), \\ Professor(Chikitsa) R.A.Podar (Govt.) Medical College, Worli, Mumbai 18
}

\section{What is Herpes zoster (Modern view)}

- Herpes zoster (shingles) is a sporadic disease that results from reactivation of latentVZV from dorsal rootganglia.

- Herpes zoster is characterized by a unilateral vesicular dermatomal eruption, often associated with severe pain. The dermatomes from T3 to L3 are most frequently involved.

- Herpes zoster occurs at all ages, but its incidence is highest (5-10 cases per 1000 persons) among individuals in the sixth decade of life andbeyond.

- Recurrent Herpes zoster is exceedingly rare except in immune compromised hosts, especially those withAIDS.

- The onset of disease is heralded by pain within the dermatome, which may precede lesions by $48-72$ hrs; an erythmatousmaculopapular rash evolves rapidly intovesicular lesions.

- In the normal host, these lesions may remain few in number and continue to form for only 3-5 days. The total duration of disease is generally 7-10 days; however, it maytake as long as 2-4 weeks for the skin to return tonormal.

What is Kaksha (Ayurvedic view)-

Symptoms of Kaksha are quite similar as that of 'Herpes zoster', which is a 'Kshudrarog' and generally affects Rasavahastrotas, Raktavahastrotas, Mamsavahastrotas and Lasikagranthi. It mainly spreads in Kaksha region [Bahu, Parshva, Ansa and kaksha] with Daha (Burning sensation) as well as Vedana (Pain) and Rakta or KrushnavarniPidika (Reddish and Blackish eruptions). It has described in classical text Madhavanidana in Kshudrarogaadhyaya under shloka no. 14.

\section{Description about the case-}

50 year old female patient came in a OPD of M.A. Podar Hospital, Worli, Mumbai with diagnosis as 'Herpes zoster' by a Allopathic physician. She was suffering from severe Herpes zoster since 15 days.

\section{Investigations-}

All routine investigations like Blood Sugar (Fasting \& Post-Prandial), Lipid Profile, LFT, RFT and CBC were within normal limits, except Blood ESR raised (40 mm/hr).

X-ray (chest) PA view and ECG also showed no major abnormalities.

\section{Ayurvedic clinical exams-}
A) AshtavidhaParikshan-
1) Nadi- $88 / \mathrm{min}$
2) Mala- shows no abnormality
3) Mutra- shows few puscells 
Dr. Parulkar Geeta D, International Journal of Ayurvedic \& Herbal Medicine 7(6) Nov.-Dec. 2017 (2932-2934)

4) Jivha- Sama(Coated)

5) Shabda- Shows noabnormality

6) Sparsha- Ruksha (withdryness)

7) Druk- shows no abnormalitiy

8) Aakruti- Madhyama(Medium)

B) VikrutaStrotasParikshan-

1) RasavahaStrotas- Strava (Secretions), Kandu(Itching)

2) RaktavahaStrotas- Raktabh-KrushnavarniVaivarnya (Reddish and Blackish Discoloration), Daha(Burningsensation)

3) MamsavahaStrotas- Raktabh-KrushnavarniPidika (Reddish and Blackisheruptions)

\section{Treatment given-}

For Internal Use (2weeks)-

i. Aarogyavardhinivati $250 \mathrm{mg} \times 3$

ii. Triphalaguggulu $250 \mathrm{mg} \times 3$

iii. Sarivadyasava $10 \mathrm{ml}+$ Khadirarishta $10 \mathrm{ml}+$ Koshnajala (Lukewarm water) $200 \mathrm{ml} \times 3$

For External Use (2weeks)-

i. Malahara (Ointment) made by mixture of

ShatadhautaGhruta 200 gms + HaridraChurna 5 gms + RaktachandanChurna 5 gms + SarivaChurna $5 \mathrm{gms}$

\section{Results-}

Follow-up taken twice a week for two weeks. Patient had shown remarkable results, which had shown below in clinical parameters changes-

\begin{tabular}{|c|c|c|}
\hline Symptoms & After First week & After Second week \\
\hline Vaivarnya(Discoloration) & $3+$ & $1+$ \\
\hline Strava (Secretions) & $3+$ & 0 \\
\hline Kandu (Itching) & $2+$ & 0 \\
\hline Daha (Burning sensation) & $3+$ & 0 \\
\hline Vedana (Pain) & $2+$ & 0 \\
\hline
\end{tabular}

\section{Clinical Parameters Gradations-}

Vaivarnya (Discoloration) - Grade 0 - Absence ofdiscoloration

Grade 1 - Mild : Faint reddish discoloration Grade 2 - Moderate : Reddish black discoloration Grade 3 Severe : Black discoloration

Strava (Secretions)-

Grade 0 - Absence of secretions

Grade 1 - Mild :Secretions occasionally

Grade 2 - Moderate : Secretions more than once, but not throughout the day

Grade 3 - Severe : Secretions throughout the day

Kandu (Itching)-

Grade 0 - Absence of Itching

Grade 1 - Mild : Itching bearable without medication also Grade 2 - Moderate : Itching bearable after medication only Grade 3 - Severe : Itching unbearable after medication also 
Dr. Parulkar Geeta D, International Journal of Ayurvedic \& Herbal Medicine 7(6) Nov.-Dec. 2017 (2932-2934)

Daha (Burning sensation)-

Grade 0 - Absence of burning sensation

Grade 1 - Mild : Burning sensation occasionally

Grade 2 - Moderate : Burning sensation more than twice but not continuously

Grade 3 - Severe : Burning sensation throughout the day

Vedana (Pain)-

Grade 0 - Absence of Pain

Grade 1 - Mild : Pain occasionally

Grade 2 - Moderate : Pain more than twice but not continuously

Grade 3 - Severe : Pain throughout the day

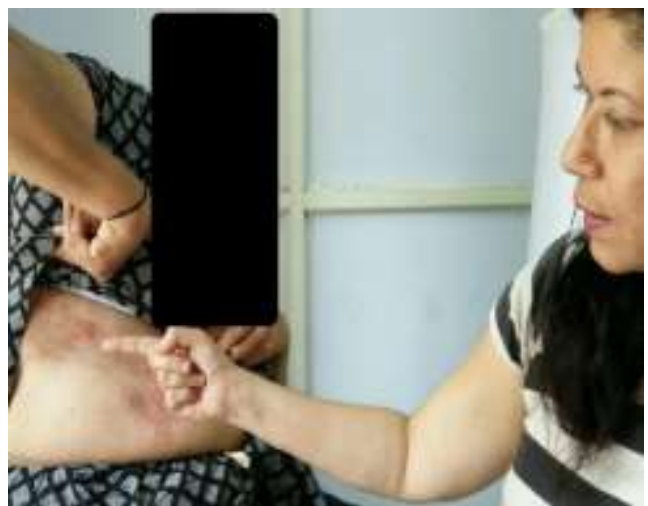

Beforetreatment

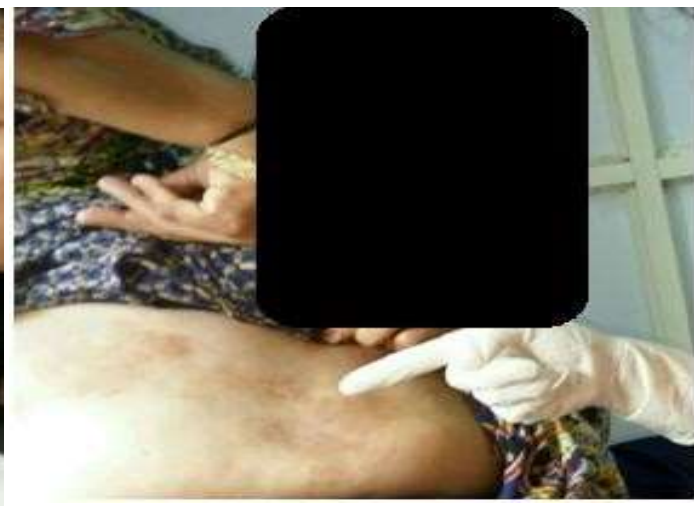

Aftertreatment

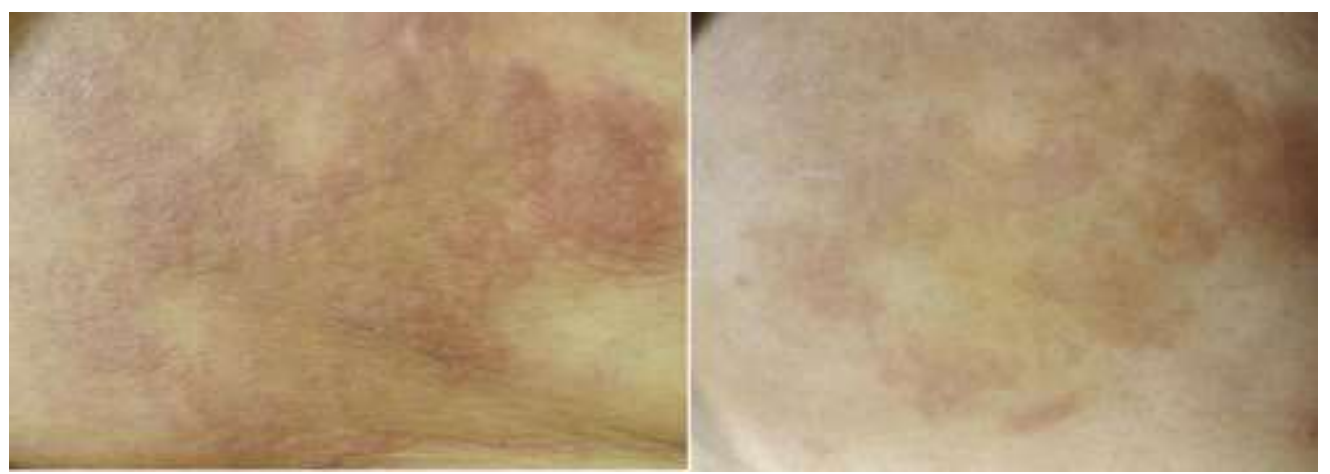

\section{Discussion with Probable mode of action-}

Aarogyavardhini is famous Ayurvedic drug for eradication of skin diseases, as well as Haritaki and Pippali among TriphalaGuggulu are useful to root out skin eruptions. Sarivadyasava and Khadirarishta are also beneficial to pacify discoloration.

ShatadhautaGhruta used as a Malahara (Ointment) is effective in reducing the inflammation. It moisturizes the skin and acts as an Astringent. The combination of Haridra, RaktaChandan and Sariva are best to control the spreading of dermal disease.

\section{Conclusion-}

Herpes zoster can be treated and relieved by Ayurvedic managemen

\section{References-}

1. Harrison's Principles of Internal Medicine, $18^{\text {th }}$ edition, Pages3059-3060.

2. Madhavanidana, Kshudrarogaadhyaya

3. Kayachikitsa, Vaidya Y.G. Joshi, 2010 edition, Published by Pune SahityaVitarana 